\title{
SSTR-Mediated Imaging in Breast Cancer: Is There a Role for Radiolabeled Somatostatin Receptor Antagonists?
}

\author{
Simone U. Dalm ${ }^{1}$, Joost Haeck ${ }^{1}$, Gabriela N. Doeswijk ${ }^{1}$, Erik de Blois ${ }^{1}$, Marion de Jong ${ }^{1}$, and Carolien H.M. van Deurzen ${ }^{2}$ \\ ${ }^{1}$ Department of Radiology and Nuclear Medicine, Erasmus MC, Rotterdam, The Netherlands; and ${ }^{2}$ Department of Pathology, \\ Erasmus MC, Rotterdam, The Netherlands
}

\begin{abstract}
Recent studies have shown enhanced tumor targeting by novel somatostatin receptor (SSTR) antagonists compared with clinically widely used agonists. However, these results have been obtained mostly in neuroendocrine tumors, and only limited data are available for cancer types with lower SSTR expression, including breast cancer (BC). To date, two studies have reported higher binding of the antagonist than the agonist in $\mathrm{BC}$, but in both studies only a limited number of cases were evaluated. In this preclinical study, we further investigated whether the application of an SSTR antagonist can improve SSTR-mediated BC imaging in a large panel of BC specimens. We also generated an in vivo BC mouse model and performed SPECT/MRI and biodistribution studies. Methods: Binding of ${ }^{111} \mathrm{In}$-DOTA-Tyr ${ }^{3}$-octreotate (SSTR agonist) and ${ }^{111} \mathrm{In}$-DOTAJR11 (SSTR antagonist) to 40 human BC specimens was compared using in vitro autoradiography. SSTR2 immunostaining was performed to confirm SSTR2 expression of the tumor cells. Furthermore, binding of the radiolabeled SSTR agonist and antagonist was analyzed in tissue material from 6 patient-derived xenografts. One patient-derived xenograft, the estrogen receptor-positive model $\mathrm{T} 126$, was chosen to generate in vivo mouse models containing orthotopic breast tumors for in vivo SPECT/MRI and biodistribution studies after injection with ${ }^{177} \mathrm{Lu}-\mathrm{DOTA}-\mathrm{Tyr}^{3}$-octreotate or ${ }^{177} \mathrm{Lu}-$ DOTA-JR11. Results: ${ }^{111}$ In-DOTA-JR11 binding to human BC tissue was significantly higher than ${ }^{111}$ In-DOTA-Tyr ${ }^{3}$-octreotate binding $(P<0.001)$. The median ratio of antagonist binding versus agonist binding was 3.39 (interquartile range, 2-5). SSTR2 immunostaining confirmed SSTR2 expression on the tumor cells. SPECT/MRI of the mouse model found better tumor visualization with the antagonist. This result was in line with the significantly higher tumor uptake of the radiolabeled antagonist than of the agonist as measured in biodistribution studies 285 min after radiotracer injection (percentage injected dose per gram of tissue: $1.92 \pm$ 0.43 vs. $0.90 \pm 0.17 ; P=0.002$ ). Conclusion: SSTR antagonists are promising candidates for $\mathrm{BC}$ imaging.
\end{abstract}

Key Words: breast cancer; imaging; somatostatin receptor; agonist; antagonist

J Nucl Med 2017; 58:1609-1614

DOI: 10.2967/jnumed.116.189035

Received Jan. 4, 2017; revision accepted Apr. 18, 2017.

For correspondence or reprints contact: Simone U. Dalm, Room Na2510, P.O. Box 2040, Erasmus MC, 3000 CA Rotterdam, The Netherlands.

E-mail: s.dalm@erasmusmc.nl

Published online Apr. 27, 2017.

COPYRIGHT (C 2017 by the Society of Nuclear Medicine and Molecular Imaging.
$\mathbf{R}$ eceptor-mediated nuclear imaging is successfully applied in patients with neuroendocrine tumors. For this purpose, radiolabeled somatostatin receptor (SSTR) agonists are used that target SSTRs overexpressed on tumor cells. In 1990, Reubi et al. (1) reported SSTR expression in $21 \%-46 \%$ of BC specimens studied by in vitro autoradiography. Since then, multiple clinical studies targeting these receptors for imaging purposes have been performed. However, because of conflicting results (sensitivity ranging from $36 \%$ to $100 \%$ and specificity ranging from $22 \%$ to $100 \%$ (2)), SSTR-mediated nuclear imaging is currently not routinely used in BC patients. Low and heterogeneous SSTR expression seems to be the main reason for unsuccessful tumor targeting, as described in our recently published review article (2). Therefore, novel developments within the field of nuclear medicine are needed to improve SSTR-mediated imaging of $\mathrm{BC}$ in order to ultimately provide a role for this method in $\mathrm{BC}$ patient care.

A promising development in the field of SSTR-mediated imaging is the application of SSTR antagonists. Enhanced tumor targeting of receptor antagonists versus receptor agonists is counterintuitive, since agonists can be internalized, leading to accumulation of radioactivity in cells. However, Ginj et al. (3) reported that SSTR antagonists bind to more binding sites than agonists. Recent studies demonstrating enhanced tumor targeting of neuroendocrine tumors with SSTR antagonists (3-7) indicate that the ability of the antagonist to bind to more binding sites may be more important than internalization of SSTR agonists. This possibility is consistent with a clinical pilot study by Wild et al. (7), who reported a 1.7-10.6 times higher tumor dose with the SSTR antagonist ${ }^{177} \mathrm{Lu}-\mathrm{DOTA}-J R 11$ than with the SSTR agonist ${ }^{177} \mathrm{Lu}$-DOTA-Tyr ${ }^{3}$-octreotate. The above-mentioned studies were based mainly on neuroendocrine tumors. However, this enhanced binding of radiolabeled SSTR antagonists may be especially interesting for targeting of cancer types with a lower SSTR expression, such as BC. Cescato et al. (4) and Reubi et al. (8) demonstrated higher binding of SSTR antagonists than of SSTR agonists to BC tissue in vitro. However, those promising results were based on evaluation of tissue from only $7 \mathrm{BCs}$ in one study and 13 in the other. Therefore, the aim of the current study was to further investigate whether the use of SSTR antagonists can provide novel possibilities for SSTR-mediated imaging of BC. To do so, binding of the radiolabeled SSTR agonist ${ }^{111}$ In-DOTA-Tyr ${ }^{3}$-octreotate to a large panel of human BC specimens was compared with that of the SSTR antagonist ${ }^{111}$ In-DOTA-JR11. Furthermore, we generated a suitable BC xenograft mouse model and performed preliminary SPECT/MRI and biodistribution studies after injection of ${ }^{177} \mathrm{Lu}$-DOTA-JR11 or ${ }^{177}$ Lu-DOTA-Tyr ${ }^{3}$-octreotate. 


\section{MATERIALS AND METHODS}

\section{Radioligands}

The SSTR agonist DOTA-Tyr ${ }^{3}$-octreotate (BioSynthema) and the SSTR antagonist DOTA-JR11 (kindly provided by Dr. Helmut Maecke), with high SSTR2 affinity, were used (Table 1). The tracers were radiolabeled with ${ }^{111} \mathrm{In}$ (Covidien) or ${ }^{177} \mathrm{Lu}$ (IDB Holland BV) as previously described (9). Specific activity was 80 and $100 \mathrm{MBq} / \mathrm{nmol}$ for the ${ }^{111}$ In- and ${ }^{177} \mathrm{Lu}$-labeled peptide analogs, respectively. Radiometal incorporation and radiochemical purity were higher than $90 \%$.

\section{Human BC Specimens}

Fresh-frozen tissue specimens from 40 human BCs (31 ductal carcinomas, 4 lobular carcinomas, and 5 other subtypes) were selected from the Erasmus MC tissue bank for autoradiography studies comparing SSTR agonist and antagonist binding. Most cases were positive for estrogen receptor (ER; 75\%) or progesterone receptor (PR; 55\%) and negative for human epidermal growth factor 2 (HER2; 18\%). The study adhered to the Code of Conduct of the Federation of Medical Scientific Societies in The Netherlands.

\section{In Vitro Autoradiography}

Fresh-frozen tissue sections $(10 \mu \mathrm{m})$ were incubated for $1.5 \mathrm{~h}$ with $100 \mu \mathrm{L}$ of $10^{-9} \mathrm{M}^{111}$ In-DOTA-Tyr ${ }^{3}$-octreotate or ${ }^{111}$ In-DOTA-JR11, with or without $10^{-6} \mathrm{M}$ octreotide as a control for receptor specificity. After incubation, the excess radiotracer was removed and tissue sections were exposed to super-resolution phosphor screens (Perkin Elmer) for $3 \mathrm{~d}$, after which the screens were read using a Cyclone phosphor imager (Perkin Elmer). Binding of the radiotracers to tumorcontaining regions, identified by hematoxylin and eosin staining of adjacent tissue sections, was quantified using OptiQuant software (Perkin Elmer) and expressed as digital light units (DLU) per square millimeter. Quantified uptake was corrected for nonspecific binding by subtracting the DLU/mm $\mathrm{mm}^{2}$ of the blocked sections from the DLU/mm ${ }^{2}$ of the unblocked sections (net DLU/mm ${ }^{2}=$ total DLU $/ \mathrm{mm}^{2}$ - blocked $\mathrm{DLU} / \mathrm{mm}^{2}$ ). Drops containing $1 \mu \mathrm{L}$ of $10^{-9} \mathrm{M}$ of the radiotracers were used as standards to determine the added dose (DLU/mm $\mathrm{mm}^{2}$ standards $\times 100$ ). Tumor-bound radiotracer was expressed relative to the added dose $(\% \mathrm{AD})$.

\section{SSTR2 Immunostaining}

SSTR2 immunostaining was performed on fresh-frozen tumor tissue, using the Benchmark Ultra automated staining system (Ventana Medical Systems) according to the manufacturer's instructions. The tissue sections were fixed for $1 \mathrm{~h}$ in neutral buffered formalin and washed in phosphate-buffered saline before immunostaining. Subsequently, antigen retrieval, immunostaining for SSTR2 (SS-8000-RM-1 [Biotrend], 1:50 dilution, $120 \mathrm{~min}$ at $36^{\circ} \mathrm{C}$ ), amplification, detection, and visualization were performed using ULTRA CC2 cell-conditioning solution, an ultraView amplification kit, and an ultraView universal DAB detection kit (all from Ventana Medical Systems). The immunostained sections were counterstained with hematoxylin. An experienced pathologist, masked to any histopathologic tumor information (e.g., ER, PR, and HER2 status) and the autoradiography results, scored the stained sections on intensity (negative, weak, moderate, or strong) and expression (homogenous vs. heterogeneous).

\section{Mouse Model, SPECT/MRI, and Biodistribution}

All animal experiments were approved by the Animal Welfare Committee and conducted in accordance with accepted guidelines.

To select an appropriate in vivo mouse model, a panel of 6 excised tumors from patient-derived xenografts with varying hormone receptor expression was autoradiographically tested for its ability to bind the agonist and the antagonist. Subsequently, one xenograft that showed high radiotracer binding was chosen to create an in vivo model for imaging and biodistribution studies. Preferably, this model should be ER-positive, since ER-positive BC seems to be the most promising subtype for SSTR-mediated imaging, in view of previously published studies demonstrating that high SSTR2 expression is associated with ER-positive BC (10-12).

For the in vivo studies, BALB/c $n u / n u$ female mice containing ERpositive patient-derived T126 xenografts were used. To generate this model, small tumor pieces $\left(\sim 6 \mathrm{~mm}^{3}\right)$ from a donor animal were transplanted into the fourth mammary fat pad of BALB/c nu/nu female mice supplemented with $\beta$-estradiol, $4 \mathrm{mg} / \mathrm{L}$. The initial generation of the model was performed as described by Marangoni et al. (13)

When the tumors were large enough $\left(\geq 200 \mathrm{~mm}^{3}\right)$, the animals received an intravenous injection $(\sim 20 \mathrm{MBq} / 200 \mathrm{pmol})$ of ${ }^{177} \mathrm{Lu}-$ DOTA-Tyr ${ }^{3}$-octreotate or ${ }^{177} \mathrm{Lu}$-DOTA-JR11 (6 animals per radiotracer). SPECT/MRI was performed $240 \mathrm{~min}$ afterward while the animals were anesthetized using isoflurane $/ \mathrm{O}_{2}$ and their body temperature was maintained. Focused SPECT images were acquired using a 4-head multipinhole SPECT/MRI system (NanoScan; Mediso Medical Imaging) in $30 \mathrm{~min}$ (28 projections; $60 \mathrm{~s}$ /projection). Images were reconstructed using ordered-subset expectation maximization with 6 iterations. Concerning MRI, T1- and T2-weighted images were acquired using a gradient echo sequence (repetition time/echo time, 12/2 $\mathrm{ms}$ ) and a spin echo sequence (repetition time/echo time, 4,500/52 $\mathrm{ms})$. The other scan parameters included a $70-\mathrm{mm}$ field of view, a $128 \times 128$ matrix, and a $1-\mathrm{mm}$ slice thickness. The MR images were used to assess tumor composition and were quantified using the scanner software (Nucline, version 3; Mediso Medical Imaging). On the T2-weighted MR images, a region of interest was drawn around the tumor that best showed the tissue boundaries. The total SPECT uptake within the region of interest was divided by the tumor volume to give a volumetric uptake $\left(\mathrm{kBq} / \mathrm{cm}^{3}\right)$ that could be compared between subjects.

After imaging (at $285 \mathrm{~min}$ after injection), the animals were euthanized and their organs and tumors excised, weighed, and counted in an automatic $\gamma$-counter (1480 Wizard; PerkinElmer) to determine radioactivity uptake. Standards containing $10 \mu \mathrm{L}$ of the injected activity were also counted in the $\gamma$-counter to determine the injected dose. Subsequently, tumor and organ uptake relative to the injected

TABLE 1

SSTR Affinity of DOTA-JR11 and DOTA-Tyr ${ }^{3}$-Octreotate

\begin{tabular}{|c|c|c|c|c|c|}
\hline Radioligand & SSTR1 & SSTR2 & SSTR3 & SSTR4 & SSTR5 \\
\hline DOTA-JR11* & $>1,000$ & $0.72 \pm 0.12$ & $>1,000$ & $>1,000$ & $>1,000$ \\
\hline DOTA-Tyr ${ }^{3}$-octreotate ${ }^{\dagger}$ & $>10,000$ & $1.5 \pm 0.4$ & $>1,000$ & $453 \pm 176$ & $547 \pm 160$ \\
\hline
\end{tabular}

${ }^{*}$ Data are from Fani et al. (14).

${ }^{\dagger}$ Data are from Reubi et al. (15). 


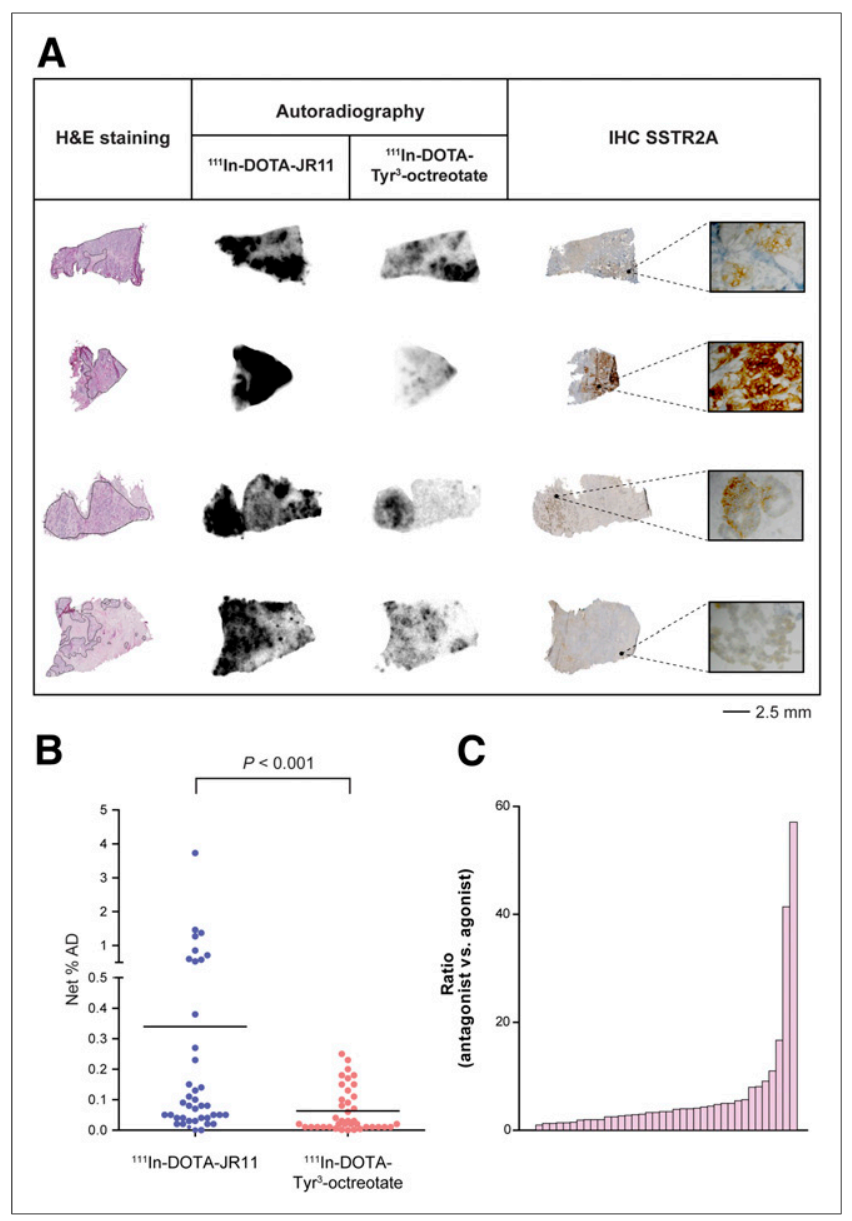

FIGURE 1. (A) Representative autoradiography results for ${ }^{111}$ In-DOTAJR11 and ${ }^{111}$ In-DOTA-Tyr ${ }^{3}$-octreotate. Hematoxylin and eosin (H\&E) staining and SSTR2 immunohistochemistry $(\mathrm{IHC})$ staining were performed on adjacent tissue sections to indicate tumor cells and SSTR2 expression, respectively. (B) Quantified uptake (net \%AD, corrected for unspecific binding) of radiotracers. (C) Ratio of radiotracer binding (antagonist to agonist).

dose were calculated and expressed as the percentage injected dose (\%ID) per gram of tissue. To determine the specificity of radiotracer uptake, an additional group of animals (2 for each radiotracer) was injected with ${ }^{177} \mathrm{Lu}$-DOTA-JR11 or ${ }^{177} \mathrm{Lu}$-DOTA-Tyr ${ }^{3}$-octreotate $(2$ $\mathrm{MBq} / 200 \mathrm{pmol}$ ) plus unlabeled DOTA-JR11 or DOTA-Tyr ${ }^{3}$-octreotate $(40 \mathrm{~nm})$, respectively, and biodistribution studies were performed at the same time point after injection $(285 \mathrm{~min})$ as for the animals that were scanned.

For the $\gamma$-counter measurements, a radionuclide-specific energy window, a counting time of $60 \mathrm{~s}$, and a counting error of less than $5 \%$ were used.

\section{Statistics}

Prism 5 software (GraphPad) was used for statistical analyses. The Wilcoxon signed rank test was used to compare the net \% AD of the radiotracers in human $\mathrm{BC}$ samples. To compare radioligand binding between samples positive for ER, PR, and HER2 and samples negative for ER, PR, and HER2, and to compare in vivo radiotracer uptake in the tumor xenografts and tumor-to-organ ratios, the Mann-Whitney test and the unpaired $t$ test were used. A $P$ value of less than 0.05 was considered statistically significant.

\section{RESULTS}

\section{In Vitro Autoradiography and SSTR2 Immunostaining of Human BC Specimens}

The results of the in vitro autoradiography experiment were quantified. The net \% AD of the antagonist bound to the $\mathrm{BC}$ specimens was significantly higher $(P<0.001)$ than that of the agonist (Fig. 1). Among the receptor-positive tumors (38/40), the ratio of $\% \mathrm{AD}$ antagonist to $\% \mathrm{AD}$ agonist ranged from 1 (only 1 case) to 57 (median, 3.39; interquartile range, 2-5).

The $\%$ AD of ${ }^{111}$ In-DOTA-JR11 and ${ }^{111}$ In-DOTA-Tyr ${ }^{3}$-octreotate showed no significant association with ER status $(P=0.2$ and 0.8$)$, PR status $(P=0.3$ and 0.1$)$, or HER2 status $(P=0.2$ and 0.09$)$.

SSTR2 immunostaining confirmed SSTR2 expression on most BC specimens (37/40) (Fig. 1A). Two specimens that were negative on immunostaining were also negative on autoradiography. One specimen was negative on SSTR2 immunostaining and positive on autoradiography but the net \%AD was low. The intensity and heterogeneity scores for the SSTR2-positive tumors are displayed in Table 2 .

\section{In Vitro Autoradiography of Patient-Derived Xenografts}

Six excised tumors from patient-derived xenografts were tested for agonist and antagonist binding (Fig. 2). Antagonist binding was clearly higher for 3 of 6 xenografts (T126, T283, and T250), whereas low binding was observed in the remaining xenografts. Two xenografts showed relatively high ${ }^{111}$ In-DOTA-JR11 and ${ }^{111}$ InDOTA-Tyr ${ }^{3}$-octreotate binding (T126 and T283) and were suited for in vivo experiments. Finally, T126 was chosen to create an in vivo mouse model.

\section{SPECT/MRI and Biodistribution Studies}

The images acquired with ${ }^{177} \mathrm{Lu}$-DOTA-Tyr ${ }^{3}$-octreotate and ${ }^{177}$ Lu-DOTA-JR11 are displayed in Figures 3A and 3B. The T1and T2-weighted MR images showed a homogeneous signal intensity throughout the tumor, indicating viability. Significantly higher radioactivity uptake on the SPECT scans was observed for ${ }^{177} \mathrm{Lu}$-DOTA-JR11 than for ${ }^{177} \mathrm{Lu}$-DOTA-Tyr ${ }^{3}$-octreotate $\left(1.12 \pm 0.4\right.$ vs. $\left.0.8 \pm 0.1 \% \mathrm{ID} / \mathrm{cm}^{3} ; P<0.05\right)$. Hematoxylin and eosin staining and SSTR2 immunostaining showed SSTR2 expression on the tumor (Figs. 3C and 3D).

In line with the SPECT/MRI results, the biodistribution studies showed that uptake in the tumor was significantly higher for the antagonist than the agonist $(P=0.022)$. High ${ }^{177}$ Lu-DOTA-JR11 and ${ }^{177} \mathrm{Lu}$-DOTA-Tyr ${ }^{3}$-octreotate uptake was also observed in the pancreas $(4.09 \pm 1.67$ and $2.57 \pm 1.64 \% \mathrm{ID} / \mathrm{g}$, respectively) and kidneys $(12.29 \pm 4.61$ and $10.06 \pm 3.42 \% \mathrm{ID} / \mathrm{g}$, respectively). There was no significant difference between ${ }^{177} \mathrm{Lu}-\mathrm{DOTA}-J R 11$ and ${ }^{177} \mathrm{Lu}$-DOTA-Tyr ${ }^{3}$-octreotate in tumor-to-pancreas ratio $(0.58 \pm 0.39$ vs. $0.55 \pm 0.39 ; P=0.82)$ or tumor-to-kidney ratio $(0.18 \pm 0.09$ vs. $0.10 \pm 0.04 ; P=0.09)$. This was also the case for the tumor-to-blood and tumor-to-muscle ratios, which were 3.0 or higher in all cases.

TABLE 2

Results of SSTR2 Immunostaining

\begin{tabular}{lrrrr}
\hline \multicolumn{1}{c}{ Finding } & Weak & Moderate & Strong & Total \\
\hline Heterogeneous & $16 \%$ & $8 \%$ & $5 \%$ & $30 \%$ \\
Homogenous & $35 \%$ & $8 \%$ & $27 \%$ & $70 \%$ \\
Total & $51 \%$ & $16 \%$ & $32 \%$ & $100 \%$ \\
\hline
\end{tabular}




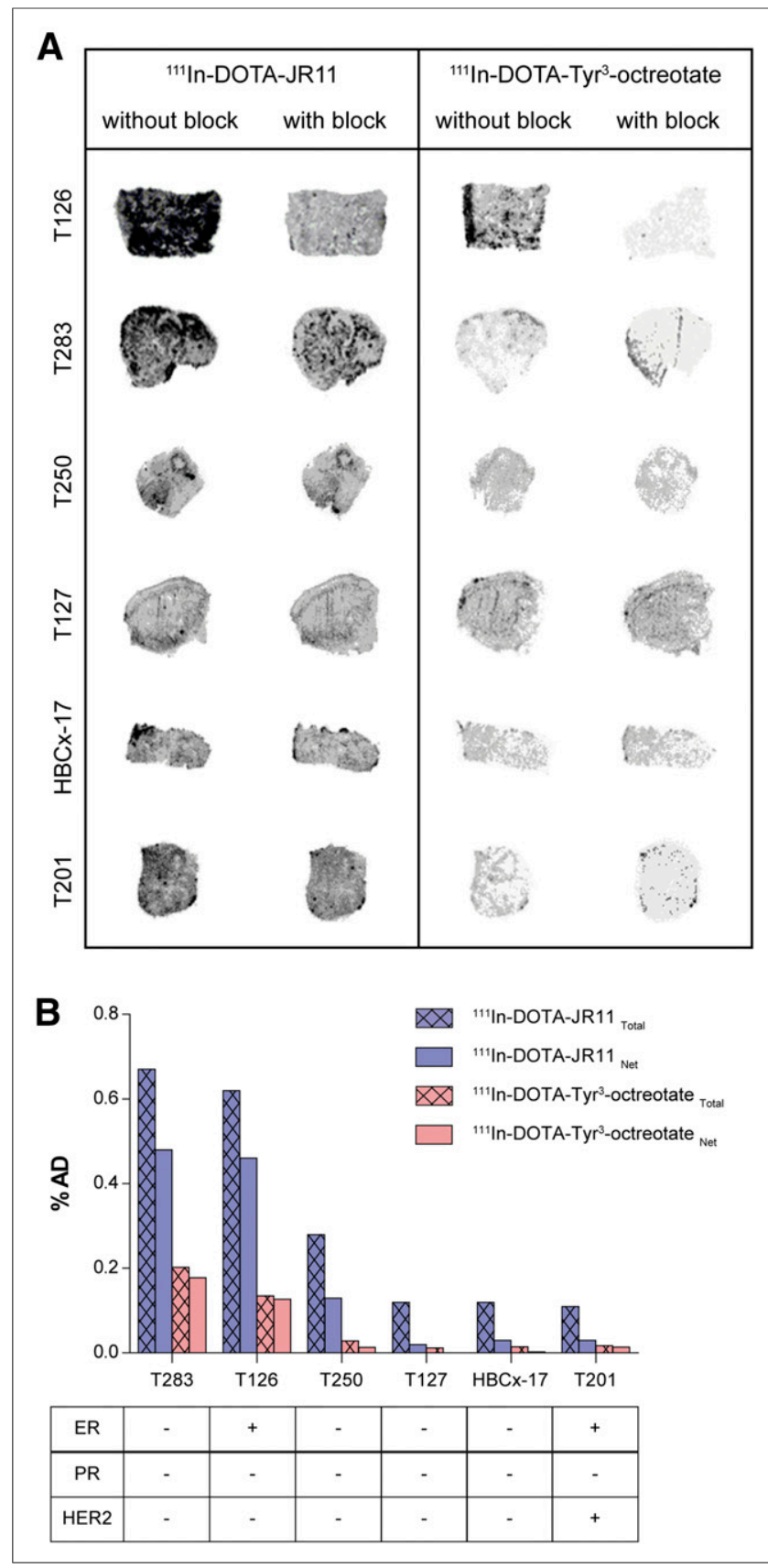

FIGURE 2. (A) Autoradiography results (without and with excess of unlabeled octreotide to determine specificity of binding) of tumor material from 6 patient-derived xenografts. Scaling was similar for agonist and antagonist binding to same patient-derived xenograft but not to different patient-derived xenografts. Therefore, uptake between different patient-derived xenografts cannot be compared on the basis of the images. Tumor sections with low radioligand binding (blocked tumor section of patient-derived xenograft T283 and T201) are color-corrected to enable visualization and may not correctly correlate with quantified uptake in B. (B) Quantified uptake (total \%AD and net \%AD corrected for unspecific binding) of ${ }^{111} \mathrm{In}$-DOTA-JR11 and ${ }^{111} \mathrm{In}$-DOTA-Tyr ${ }^{3}$ octreotate for autoradiography in A, with ER, PR, and HER2 status of patient-derived xenograft models.

Injection of ${ }^{177}$ Lu-DOTA-JR11 plus an excess of unlabeled DOTA-JR11, and of ${ }^{177} \mathrm{Lu}$-DOTA-Tyr ${ }^{3}$-octreotate plus an excess of unlabeled DOTA-Tyr ${ }^{3}$-octreotate, significantly blocked tumor uptake, demonstrating the SSTR specificity of radiotracer uptake in the tumor.

The biodistribution results and tumor-to-organ ratios are displayed in Figure 4.

\section{DISCUSSION}

SSTR-mediated nuclear imaging is currently not applied for BC because of the variable results of clinical studies. However, most of those studies were performed over a decade ago and there have recently been major improvements such as the development of SSTR antagonists. Previous research demonstrated that SSTR antagonists bind to more binding sites than agonists do (3). In BC, low SSTR expression and heterogeneous SSTR expression seem to be limiting factors in the success of SSTR-mediated nuclear imaging (2). Therefore, application of SSTR antagonists may offer novel possibilities for successful BC imaging. In the current study, we compared the SSTR agonist ${ }^{111} \mathrm{In}$-DOTA-Tyr ${ }^{3}$-octreotate and the SSTR antagonist ${ }^{111}$ In-DOTA-JR11 in 40 human BC specimens and in a BC mouse model. Since both DOTA-Tyr ${ }^{3}$-octreotate and DOTA-JR11 have high affinity for SSTR2 and low affinity for

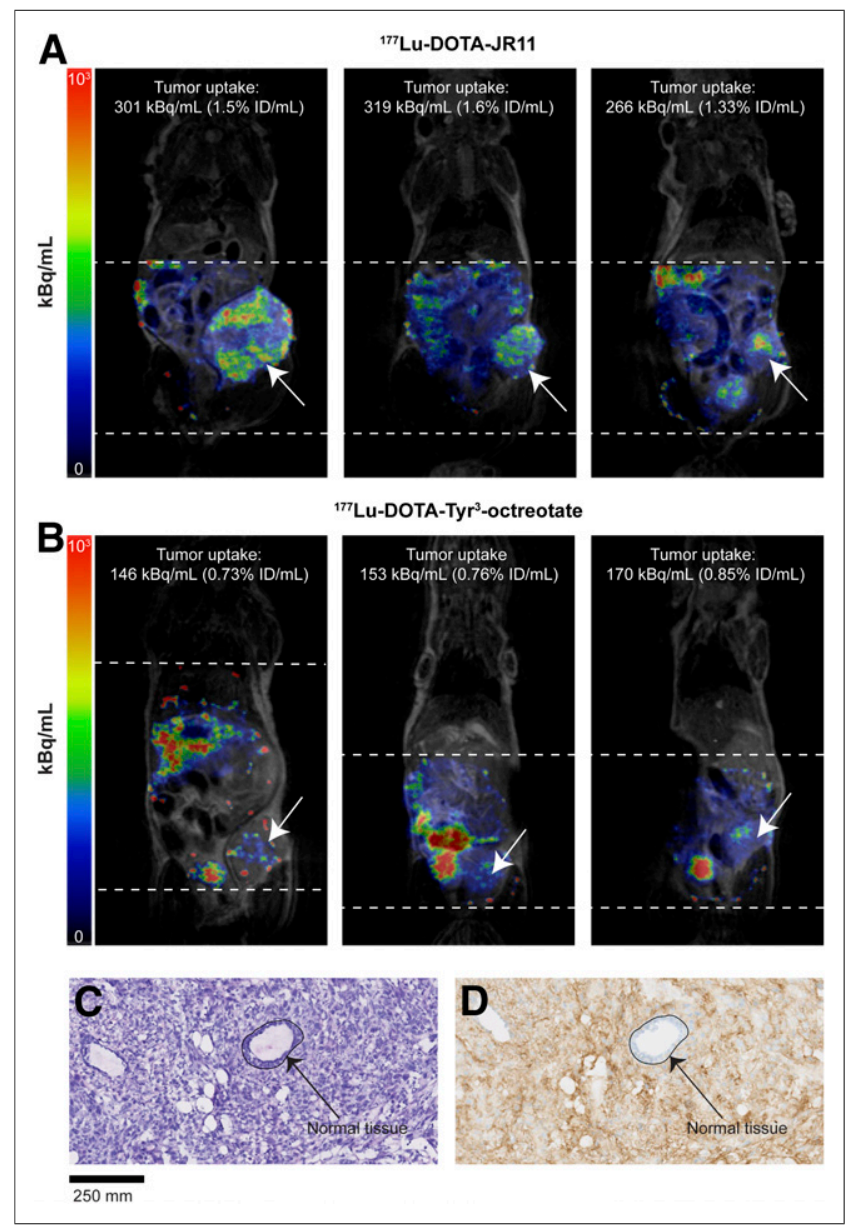

FIGURE 3. (A and B) SPECT/MR images and quantified tumor uptake after injection of ${ }^{177}$ Lu-DOTA-JR11 (A) and ${ }^{177}$ Lu-DOTA-Tyr ${ }^{3}$-octreotate (B). Scales are equal for all images. Dotted lines indicate area scanned with SPECT. Arrows indicate tumor xenografts. (C and D) Hematoxylin and eosin staining (C) and SSTR2 immunostaining (D) of excised tumors showing SSTR2 expression. Arrows indicate normal tissue (epithelial lining of duct) without SSTR2 expression. 


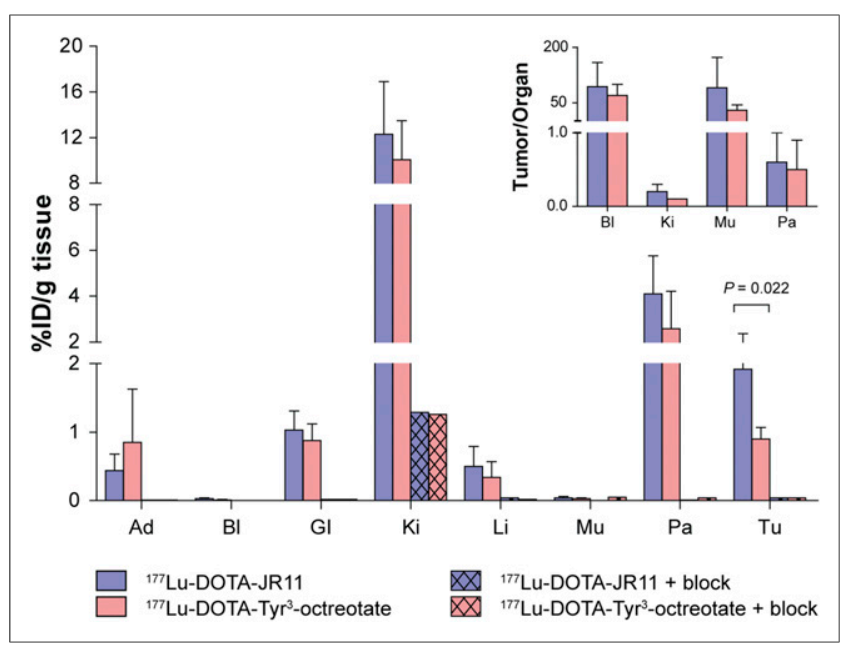

FIGURE 4. Radioactivity uptake and tumor-to-organ ratios in excised tumors and organs after imaging studies. Blocking studies were performed by injection of radiotracers plus excess of unlabeled peptide analogs. Ad = adrenals; $\mathrm{Bl}=$ blood; $\mathrm{Gl}=$ gastrointestinal tract; $\mathrm{Ki}=$ kidney; $\mathrm{Li}=$ liver; $\mathrm{Mu}=$ muscle; $\mathrm{Pa}=$ pancreas; $\mathrm{Tu}=$ tumor.

other SSTR subtypes (Table 1), the observed difference in binding and uptake is based mainly on interaction with $\operatorname{SSTR} 2(14,15)$.

We found significantly higher binding (up to 57 times higher) of ${ }^{111}$ In-DOTA-JR11 than of ${ }^{111}$ In-DOTA-Tyr ${ }^{3}$-octreotate to the BC specimens. Our findings are in line with those of Cescato et al. (4), who demonstrated higher binding of ${ }^{177} \mathrm{Lu}$-DOTA-BASS, another SSTR antagonist, than of ${ }^{177}$ Lu-DOTA-Tyr ${ }^{3}$-octreotate in 7 BCs analyzed by in vitro autoradiography. In the same study, the authors also compared binding of ${ }^{177} \mathrm{Lu}$-DOTA-BASS with that of ${ }^{177} \mathrm{Lu}$-DOTA-Tyr ${ }^{3}$-octreotate to other tumor types, including pheochromocytomas. Binding of the antagonist to BCs was as high as binding of the agonist to pheochromocytomas. Since pheochromocytomas are clinically successfully targeted with the agonist (4), there is potential for targeting of BC with SSTR antagonists. In a recent study, Reubi et al. (8) compared binding of ${ }^{125}$ I-JR11 with that of ${ }^{125} \mathrm{I}_{-} \mathrm{Tyr}^{3}$-octreotide in $\mathrm{BC}$ and also found enhanced binding of the antagonist versus the agonist. In that study, the authors also compared binding of the radioligands to neuroendocrine tumors, but under different experimental conditions, hampering comparison with results obtained in breast tumors.

In our autoradiography studies, a higher level of unspecific binding was observed for ${ }^{111} \mathrm{In}$-DOTA-JR 11 than for ${ }^{111}$ In-DOTA$\mathrm{Tyr}^{3}$-octreotate. This finding might be due to the fact that blocking studies were performed with unlabeled octreotide, a well-known SSTR agonist, rather than with an antagonist. In the in vivo blocking studies, blocking with an excess of unlabeled DOTA-JR11 demonstrated that radiolabeled JR11 is highly specific, as was also demonstrated by Reubi et al. (8) in an autoradiography experiment using ${ }^{125}$ I-DOTA-JR11 with unlabeled DOTA-JR11 added for blocking.

The group of BCs that we studied was heterogeneous. However, concerning subtype, most of the tumors were classified as ductal, ER-positive, HER2-negative carcinomas. Although this classification is consistent with the distribution of BC subtypes in the clinic, it limits the possibility of comparing SSTR expressionand SSTR agonist and antagonist binding-across different BC subtypes, because the subgroups were too small for reliable conclusions. The size of the subgroups probably also explains why we found no significant association between ER, PR, or HER2 status and ${ }^{111} \mathrm{In}$-DOTA-octreotate or ${ }^{111} \mathrm{In}$-DOTA-JR11 binding in this study, whereas we found ER and PR to have a significant positive association with SSTR expression in a previous study analyzing the receptor expression of 684 lymph node-negative BCs (12).

Immunostaining confirmed SSTR2 expression on the tumor sections analyzed by in vitro autoradiography. Most of the specimens $(51.4 \%)$ showed weak SSTR2 expression, which is in line with previous findings (2). There was a discrepancy in a single BC specimen that was negative for SSTR2 immunostaining and positive in the autoradiography experiments; however, the net \% AD was low, as might be explained by variability of SSTR2 expression within a tumor. In all other cases, SSTR2 expression on immunostaining matched the autoradiography results.

In vivo imaging was successful with both ${ }^{177} \mathrm{Lu}$-DOTA-JR11 and ${ }^{177} \mathrm{Lu}$-DOTA-Tyr ${ }^{3}$-octreotate, although tumors were visualized better with ${ }^{177} \mathrm{Lu}$-DOTA-JR11. In line with this result, the measured ex vivo tumor uptake of ${ }^{177} \mathrm{Lu}$-DOTA-JR11 was $100 \%$ higher than that of ${ }^{177} \mathrm{Lu}$-DOTA-Tyr ${ }^{3}$-octreotate, resulting in better tumor visualization. Considering that ${ }^{177} \mathrm{Lu}$ is a therapeutic radionuclide and that enhanced tumor targeting with ${ }^{177} \mathrm{Lu}$-DOTA-JR11 has been reported, the next step would be to determine whether ${ }^{177} \mathrm{Lu}-$ DOTA-JR11 can also be used for safe and effective treatment of these tumors. In line with the findings of our previous study (6) comparing the application of ${ }^{177} \mathrm{Lu}-\mathrm{DOTA}-\mathrm{Tyr}^{3}$-octreotate and ${ }^{177} \mathrm{Lu}-\mathrm{DOTA}-$ JR11 for therapeutic purposes in a neuroendocrine mouse model, we expect that the enhanced in vivo tumor uptake of the antagonist in BC will also result in better therapeutic efficacy. Thus, SSTRmediated targeting of BC might offer not only imaging possibilities (including therapy response assessment and visualization of metastases) but also therapy options.

When the radiolabeled antagonist will be used for therapy, uptake of the radiotracer in nontarget organs should be considered. Next to the tumor, high radioactivity uptake was also seen in the kidneys. However, kidney uptake can be reduced remarkably by coinjecting the radiotracers with a kidney protector such as modified fluid gelatin or a solution containing arginine and lysine $(16,17)$. Wild et al. (7) reported a 6.2 times higher tumor-to-kidney ratio for ${ }^{177} \mathrm{Lu}-$ DOTA-JR11 than for ${ }^{177} \mathrm{Lu}-\mathrm{DOTA}-\mathrm{Tyr}^{3}$-octreotate. In that study, patients were preinjected with a solution of arginine and lysine, whereas in our preclinical in vivo studies we did not apply kidney protection and showed a similar tumor-to-kidney ratio for both the antagonist and the agonist. In view of the results of Wild et al. (7), we expect that applying a kidney protector would positively influence the tumor-to-kidney ratio and that this effect would be more in favor of the antagonist because of its higher ${ }^{177}$ Lu-DOTA-JR11 uptake in the tumor.

Next to the kidneys, high uptake was also observed in the pancreas. However, the pancreas has relatively low radiosensitivity (18), and our previous study (6) showed that with the antagonist, washout is relatively faster from the pancreas than from the tumor (clearance half-life of $13.6 \mathrm{~h}$ from pancreas vs. $30 \mathrm{~h}$ from tumor xenograft as measured in mice xenografted with H69 small cell lung cancer cells). Furthermore, the pancreas was not found to be a dose-limiting organ in studies using the agonist (19).

Recently, results were published for the NETTER-1 trial (20), a large phase 3 clinical trial using ${ }^{177} \mathrm{Lu}$-DOTA-Tyr ${ }^{3}$-octreotate for treatment of midgut neuroendocrine tumors. This trial reported the side effect of grade 3 or 4 hematologic toxicity in, however, only a small percentage of the patients, and when ${ }^{177} \mathrm{Lu}-\mathrm{DOTA}-\mathrm{Tyr}^{3}$ octreotate was administered concomitantly with a renal protective agent there was no reported renal toxicity. Even though we found 
no significant difference in tumor-to-blood or tumor-to-kidney ratios between the antagonist and the agonist, the higher absolute uptake of the antagonist in background organs can have potential consequences and should be kept in mind when the radioligand is applied for therapeutic purposes. Furthermore, dosimetry studies are needed to determine whether the absolute uptake of ${ }^{177}$ Lu-DOTAJR11 in BC patients is sufficient for peptide receptor radionuclide therapy using SSTR radioligands, keeping in mind the dose-limiting organs (kidneys and bone marrow (19)).

The heterogeneous expression of SSTR2 in BC is also something that should be kept in mind, especially when the radioligand is used for therapeutic purposes. With respect to this consideration, the radionuclide that is to be coupled to the tracers should be selected carefully. If the antagonist is coupled to a radionuclide with a good penetration range (depending on the tumor size and the level of heterogeneity, ${ }^{90} \mathrm{Y}$ might be more favorable than ${ }^{177} \mathrm{Lu}$ because of its deeper penetration range), it is expected that radiation from radioligands bound to SSTR-positive BC cells can also affect neighboring cells with low or no STTR2 expression. Furthermore, imaging studies performed before therapy can determine the heterogeneity of SSTR2 expression in tumors. In addition, therapy with the antagonist might potentially be used to shrink the tumor-even heterogeneous tumors-after which other therapies could be applied.

In a study by Dude et al. (21), uptake of 2 SSTR agonists (DOTATOC and DOTA-Tyr ${ }^{3}$-octreotate) and 1 SSTR antagonist (NODAGA-JR11) was compared in a BC xenograft model (ZR751). The authors reported a higher uptake of ${ }^{68} \mathrm{Ga}$-DOTATOC than of ${ }^{68} \mathrm{Ga}-N O D A G A-J R 11$, whereas uptake of ${ }^{68} \mathrm{Ga}-N O D A G A-$ JR11 was higher than that of ${ }^{68} \mathrm{Ga}$-DOTA-Tyr ${ }^{3}$-octreotate, as is in line with our findings. This report indicates that SSTR antagonists may not always be superior to agonists for tumor targeting, as might be explained by the fact that the affinity of DOTATOC for SSTR subtypes is different from that of NODAGA-JR11 and DOTA-Tyr ${ }^{3}$-octreotate. Additional studies are needed to determine the role of SSTR subtype expression in order to choose the optimal radiotracer for targeting of $\mathrm{BC}$.

\section{CONCLUSION}

We demonstrated the advantage of radiolabeled SSTR antagonists over agonists for BC targeting in a preclinical setting, shedding new light on SSTR-mediated BC imaging. The application of SSTR antagonists, combined with recent developments such as dedicated breast cameras (22) and PET radionuclides (23), is promising and may increase the importance of SSTR-mediated imaging and treatment of $\mathrm{BC}$ patients.

\section{DISCLOSURE}

No potential conflict of interest relevant to this article was reported.

\section{REFERENCES}

1. Reubi JC, Waser B, Foekens JA, Klijn JG, Lamberts SW, Laissue J. Somatostatin receptor incidence and distribution in breast cancer using receptor autoradiography: relationship to EGF receptors. Int J Cancer. 1990;46:416-420.
2. Dalm SU, Melis M, Emmering J, Kwekkeboom DJ, de Jong M. Breast cancer imaging using radiolabelled somatostatin analogues. Nucl Med Biol. 2016;43: $559-565$.

3. Ginj M, Zhang H, Waser B, et al. Radiolabeled somatostatin receptor antagonists are preferable to agonists for in vivo peptide receptor targeting of tumors. Proc Natl Acad Sci USA. 2006;103:16436-16441.

4. Cescato R, Waser B, Fani M, Reubi JC. Evaluation of ${ }^{177} \mathrm{Lu}-\mathrm{DOTA}-\mathrm{sst} 2$ antagonist versus ${ }^{177} \mathrm{Lu}$-DOTA-sst2 agonist binding in human cancers in vitro. $\mathrm{J}$ Nucl Med. 2011;52:1886-1890.

5. Wild D, Fani M, Behe M, et al. First clinical evidence that imaging with somatostatin receptor antagonists is feasible. J Nucl Med. 2011;52:14121417.

6. Dalm SU, Nonnekens J, Doeswijk GN, et al. Comparison of the therapeutic response to treatment with a ${ }^{177} \mathrm{Lu}$-labeled somatostatin receptor agonist and antagonist in preclinical models. J Nucl Med. 2016;57:260-265.

7. Wild D, Fani M, Fischer R, et al. Comparison of somatostatin receptor agonist and antagonist for peptide receptor radionuclide therapy: a pilot study. $\mathrm{J} \mathrm{Nucl}$ Med. 2014;55:1248-1252.

8. Reubi JC, Waser B, Macke H, Rivier J. Highly increased ${ }^{125}$ I-JR11 antagonist binding in vitro reveals novel indications for sst 2 targeting in human cancers. J Nucl Med. 2017;58:300-306.

9. de Blois E, Chan HS, de Zanger R, Konijnenberg M, Breeman WA. Application of single-vial ready-for-use formulation of ${ }^{111} \mathrm{In}$ - or ${ }^{177} \mathrm{Lu}$-labelled somatostatin analogs. Appl Radiat Isot. 2014;85:28-33.

10. Van Den Bossche B, D'Haeninck E, De Vos F, et al. Oestrogen-mediated regulation of somatostatin receptor expression in human breast cancer cell lines assessed with ${ }^{99 \mathrm{~m}}$ Tc-depreotide. Eur J Nucl Med Mol Imaging. 2004;31:10221030 .

11. Kumar U, Grigorakis SI, Watt HL, et al. Somatostatin receptors in primary human breast cancer: quantitative analysis of mRNA for subtypes 1-5 and correlation with receptor protein expression and tumor pathology. Breast Cancer Res Treat. 2005;92:175-186.

12. Dalm SU, Sieuwerts AM, Look MP, et al. Clinical relevance of targeting the gastrinreleasing peptide receptor, somatostatin receptor 2 , or chemokine c-X-c motif receptor 4 in breast cancer for imaging and therapy. J Nucl Med. 2015;56:1487-1493.

13. Marangoni E, Vincent-Salomon A, Auger N, et al. A new model of patient tumor-derived breast cancer xenografts for preclinical assays. Clin Cancer Res. 2007;13:3989-3998.

14. Fani M, Braun F, Waser B, et al. Unexpected sensitivity of sst2 antagonists to N-terminal radiometal modifications. J Nucl Med. 2012;53:1481-1489.

15. Reubi JC, Schar JC, Waser B, et al. Affinity profiles for human somatostatin receptor subtypes SST1-SST5 of somatostatin radiotracers selected for scintigraphic and radiotherapeutic use. Eur J Nucl Med. 2000;27:273-282.

16. van Eerd JE, Vegt E, Wetzels JF, et al. Gelatin-based plasma expander effectively reduces renal uptake of ${ }^{111} \mathrm{In}$-octreotide in mice and rats. J Nucl Med. 2006; 47:528-533.

17. Rolleman EJ, Valkema R, de Jong M, Kooij PP, Krenning EP. Safe and effective inhibition of renal uptake of radiolabelled octreotide by a combination of lysine and arginine. Eur J Nucl Med Mol Imaging. 2003;30:9-15.

18. Stewart FA, Akleyev AV, Hauer-Jensen M, et al. ICRP publication 118: ICRP statement on tissue reactions and early and late effects of radiation in normal tissues and organs - threshold doses for tissue reactions in a radiation protection context. Ann ICRP. 2012;41:1-322.

19. de Jong M, Krenning E. New advances in peptide receptor radionuclide therapy. J Nucl Med. 2002;43:617-620.

20. Strosberg J, El-Haddad G, Wolin E, et al. Phase 3 trial of ${ }^{177} \mathrm{Lu}$-dotatate for midgut neuroendocrine tumors. N Engl J Med. 2017;376:125-135.

21. Dude I, Zhang Z, Hundal N, et al. Preclinical evaluation of somatostatin receptor agonist versus antagonist radioligands for breast cancer imaging [abstract]. J Nucl Med. 2016;57(suppl 2):1175.

22. Vercher-Conejero JL, Pelegri-Martinez L, Lopez-Aznar D, Cozar-Santiago Mdel P. Positron emission tomography in breast cancer. Diagnostics (Basel). 2015;5:61-83.

23. Buchmann I, Henze M, Engelbrecht S, et al. Comparison of ${ }^{68}$ Ga-DOTATOC PET and ${ }^{111}$ In-DTPAOC (Octreoscan) SPECT in patients with neuroendocrine tumours. Eur J Nucl Med Mol Imaging. 2007;34:1617-1626. 